\title{
The Theory of the Development of an Electromagnetic Engine for Automotive Use
}

\author{
Ataur Rahman ${ }^{1 *}$, Mizanur Rahman², Hafizzul Karim ${ }^{1}$ \\ ${ }^{1}$ Department of Mechanical Engineering, International Islamic University, Malaysia50728 KL, Malaysia \\ ${ }^{2}$ Department of Mechatronics Engineering, International Islamic University, Malaysia50728 KL, Malaysia
}

Received: February 07, 2017; Accepted: February 23 , 2017; Published: March 20, 2017

*Corresponding author: Ataur Rahman, Department of Mechanical Engineering, International Islamic University, Malaysia50728 KL, Malaysia,

E-mail: arat@iium.edu.my

\begin{abstract}
The need to reduce the emission emitted from IC engine powered vehicle is paramount, as Malaysia has pledged to reduce $40 \%$ of $\mathrm{CO}_{2}$ intensity by 2020 from 2005 level by $25 \%$ improvement in average fuel consumption. The introduction of Electromagnetic Engine (EE) is one of the initiatives, however the electromagnetic engine could face several weaknesses amongst which are: limited driving range, thermal control, charging infrastructure, and overall limited efficiency. This study presents an electromagnetic propulsion system for automotive use that has a total different operating sequence compared to IC engines. It works by the principle of magnetic attraction and repulsion between electromagnet and a permanent magnet. The power supply interruption made by means of the reed switch to move down the piston. Thus, the back-and-forth movement of the piston occurs without failure. The experiment has been conducted on the determination of the electromagnetic engine performance by varying the applied loads on the crankshaft. The efficiency has been found of the model EME in the range of $12-20 \%$. However, it could be achieved about $40-45 \%$ by proper sealing the magnetic flux of the electromagnet and alignment, exact fitting the piston and cylinder and prevent the magnetic flux leaking and heat generation, and the development of piston and cylinder with the aluminium alloy and metallic liner.
\end{abstract}

Keywords: Electromagnetic engine; Leed switching mechanism; Emission; Propulsion

\section{Introduction}

Electromagnetic Propulsion (EMP) is the principle of accelerating an object by the utilization of a flowing electrical current and magnetic fields. The electrical current is used to either create an opposing magnetic field, or to charge a field, which can then be repelled. When a current flows through a conductor in a magnetic field, an electromagnetic force known as a Lorentz force, pushes the conductor in a direction perpendicular to the conductor and the magnetic field. This repulsing force is what causes propulsion in a system designed to take advantage of the phenomenon. The term Electromagnetic Propulsion (EMP) can be described by its individual components: electromagneticusing electricity to create a magnetic field (electromagnetism), and propulsion- the process of propelling something. One key difference between EMP and propulsion achieved by electric motors is that the electrical energy used for EMP is not used to produce rotational energy for motion; though both use magnetic fields and a flowing electrical current. The science of electromagnetic propulsion does not have origins with any one individual and has application in many different fields. The thought of using magnets for propulsion continues to this day and has been dreamed [14]. In a maglev train the primary magnetic coil assembly lies below the reaction plate. There is a $1-10 \mathrm{~cm}$ air gap between that eliminates friction, allowing for speeds up to $500 \mathrm{~km} / \mathrm{h}$ [2]. An alternating electric current is supplied to the coils, which creates a change in polarity of the magnetic field. This pulls the train forward from the front, and thrusts the train forward from the back [3]. Maglev trains are promoted for their energy efficiency since they run on electricity, which can be produced by coal, nuclear, hydro, fusion, wind or solar power without requiring oil [4]. A linear induction motor consists of two parts: the primary coil assembly and the reaction plate. The primary coil assembly consists of phase windings surrounded by steel laminations, and includes a thermal sensor within a thermal epoxy $[4,5,6]$. Functioning of a linear induction motor begins with an $\mathrm{AC}$ force that is supplied to the coil windings within the primary coil assembly. This creates a traveling magnetic field that induces a current in the reaction plate, which then creates its own magnetic field. The magnetic fields in the primary coil assembly and reaction plate alternate, which generates force and direct linear motion $[8,10,1]$.

An electromagnetic engine is an engine device that is powered by the magnetic components that helps and support in the operation of reciprocating piston to propel the engine. This mechanism is achieved by attaching the device individually to the pistons giving a result to the pistons to perform the backand-forth thrusts motion. By implementing this concept in mobilizing the engine, which is zero-fuel operation, it eliminates the necessity of fuel hence preventing the environment to be polluted by the fuel residual. This engine has been designed with magnetic shielding safety components to avoid any harm to the 
people and other electronic devices from strong and rare earth magnets and electromagnets.

Fossil fuels have been a demand to the society and people of the world and it keeps on an increased pattern through the years. There will be a period of time the world has to depend on electricity as the only source 'fuel' in operating vehicbles and machinery systems. According to [9], the invention of electric vehicle has made with battery power pack, which makes the vehicle heavier. It also has lesser efficiency when compared to ICE. While, electromagnetic engines can promise an alternative to those problems due to the following reasons: Fuel and money saving, no hazardous for the environment, cost effective, low maintenance required, noise pollution reduction, lighter engine weight compared to ICE. However, there are also the disadvantages of this type of engine. Firstly, this electromagnetic engine cannot produce power as same as IC engines which are producing very high value of power. The torque at the crankshaft is also having less uniformity when compared to existing IC engines. Furthermore, the recharge centre for the battery that is used in this vehicle is developed yet in world-wide. The driving range is also not too long as same as the speed that cannot reach high velocity. For cities that have shortage of electric power, it becomes another limitation of implementing this engine to the society. The development of Electric Vehicle (EV) and Hybrid Electric Vehicle (HEV) are increasing from a year to another, the problem still persists. For example, the battery needed to operate an EV is expensive. The manufacturers or developers have not commercialised the charging centre for electric vehicle yet. The main reason for this is because there are not many people can afford to buy electric vehicle as the cost of owning an electric vehicle is very high compared with ICE cars.

The aim of this study is to develop an electromagnetic engine that can replace the Internal Combustion Engine (ICE). The electromagnetic engine needs the battery pack to power the engine propulsion. However, it needs smaller size and lower capacity of the battery, which will reduce the cost of vehicle operation and zero emission. The main challenge in completing this study is to develop magnetic force that can give optimum power to the operation of the piston at high velocity. While, the placement of the permanent magnet that will react with the Reed switch to make the smooth operation is also considered as an important issue. In addition, the challenges of this study which could make the study of electromagnetic engine or propulsion system to enhance the its efficiency.

\section{Methodology}

\section{Vehicle Propulsion Power}

The acceleration of a vehicle is determined by all the forces applied on it and represent as, $a=F_{t}-\sum F_{m} / m$, $\mathrm{m}$ is the overall mass of the vehicle, $\mathrm{F}_{\mathrm{t}}$ is the total traction force to the vehicle, and $\sum F_{m}=f_{r} W+0.5 \rho_{a} C_{D} A_{f^{v}}+W \sin \theta$ is the total resistive force. The traction force, $F_{t}$ can be estimated as follows,

\section{(i) Reduction mode:}

$$
F_{t(r m)}=\mu m g\left(\frac{L_{f}+f_{r} h / L_{w}}{1+\mu h / L_{w}}\right)
$$

where $\mathrm{m}_{\mathrm{a}}$ is the mass of the vehicle in $\mathrm{kg}$, $\mathrm{g}$ is the gravitational acceleration constant equal to $9.81 \mathrm{~m} / \mathrm{s}^{2}, \mu$ the adhesion coefficient of the road, $\mathrm{L}_{\mathrm{f}}$ is the distance from the front wheel to $C G$ in $m, f_{r}$ the coefficient of rolling motion resistance, $h$ is the height of CG in $\mathrm{m}, \mathrm{L}_{\mathrm{w}}$ the wheel base in $\mathrm{m}, \theta_{\mathrm{g}}$ the slope angle with respect to the horizon in degree.

\section{(ii) Dynamic mode (moderate speed):}

The major external forces acting on a vehicle during motion are the aerodynamic resistance $R_{a}=0.5 C_{D} A_{f} V^{2}$, rolling resistance, $R_{r}=f_{r} W$, grade resistance, $R_{g}=m g \sin \theta$, the traction force at moving condition is estimated as,

$$
F_{t(d m)}=m a+f_{r} m g+m g \sin \theta+0.5 \rho_{a} C_{D} A_{f} V^{2}
$$

where, $\rho_{\text {air }}$ is the air density in $\mathrm{kg} / \mathrm{m}^{3}, \mathrm{C}_{\mathrm{D}}$ is the coefficient of aerodynamic resistance, $\mathrm{A}_{\mathrm{f}}$ is the frontal area of the vehicle in $\mathrm{m}^{2}$ and $v$ is the travelling speed of the vehicle relative to the wind in $\mathrm{m} / \mathrm{s}$. Vehicle traction on the maximum grade is mainly due to the rolling resistance and grade resistance. The aerodynamic resistance, $\mathrm{R}_{\mathrm{a}}$ is considered zero as the vehicle moves on the maximum grades very slow.

Figures 1 and 2 shows the kinematice simulation for a vehicle of 5-speed manual transmission by using the MATLAB follows the program [7]. It shows that the excessive traction forces at the points of engine maximum speeds for the vehicle vehicle reduction speed for $1^{\text {st }}$ gear. The traction force also needs more for the vehicle in $2^{\text {nd }}$ and $3^{\text {rd }}$ gear which can be justified for the Eqs. 2 and 3 in case of vehicle moderate driveThis also can be supported by equation [1] for reduction drive. These points are normally defined by kinematic limit points in which gear needs to be shifted for manual, automatic adjusted for automatic and

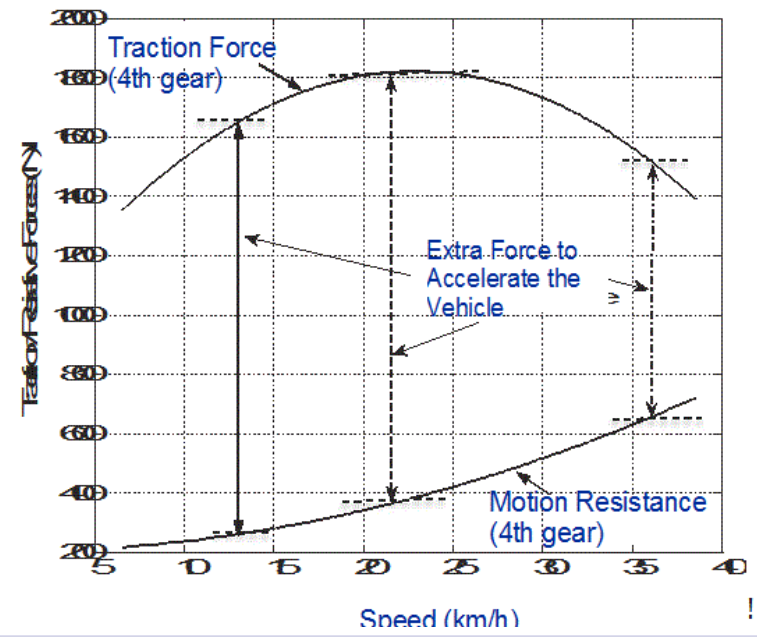

Figure 1: Vehicle acceleration. 
Continuously Variable Transmission (CVT). In cruising speed or overdirive for gear 5 , the aerodynamic motion resistance grows rapidly due to the speed of the vehicle against the air force. The traction force of the vehicle is met the total motion resistance and vehicle attains its maximum speed. The point where the vehicle traction force meets the vehicle motion resistance is called the dynamic balance point. The tractive force,

$$
V_{\text {max }}=\sqrt{\frac{2\left(F_{t}-f_{r} m g\right)}{\rho_{a} C_{D} A_{f}}} \text { where, } \mathrm{F}_{\mathrm{t}} \text { is the traction forcefor } 5^{\text {th }} \text { gear }
$$

and the extra forces need to accelerate the vehicle for the varyng time, $\frac{\partial v}{\partial t}=\frac{\partial F_{t}(t)}{m}$. The propulsion power required to propel the vehicle at maximum speed, $P_{\text {propulsion }(r m)}=F_{t(r m)}{ }^{*} V_{a c c}$; $P_{\text {propulsion }(d m)}=F_{t(d m)}{ }^{*} V_{m} ; \quad P_{\text {propulsion }(c r)}=F_{t(c r)}{ }^{*} V_{\text {max }}$. By considering the car traction at the starting of $0.5 \mathrm{~km} / \mathrm{h}$, urban speed of $40 \mathrm{~km} / \mathrm{h}$, and maximum speed of $90 \mathrm{~km} / \mathrm{h}(25 \mathrm{~m} / \mathrm{s})$, the adhesion coefficient value $\mu$ is 0.4 , coefficient of rolling motion resistance, $\mathrm{f}_{\mathrm{r}}$ is 0.02 , drag coefficient, $\mathrm{C}_{\mathrm{D}}$ is 0.24 , Frontal area, $\mathrm{A}_{\mathrm{f}}$ of $2.86 \mathrm{~m}^{2}$, and air density equals to $1.2 \mathrm{~kg} / \mathrm{m}^{3}$. The traction force the electromagnetic propulsion system needs to develop as,

$$
F_{e m}=\frac{1}{N_{g} N_{d} \eta_{t}}\left(F_{t}\right)
$$

where, $N_{g}$ is the gear ratio, $N_{d}$ is the drive ratio, $\eta_{t}$ transmission efficiency, $F_{t}$ is the traction force of the vehicle develops at tireroad interfaces. The propulsion power requirement can be claculated: for $1^{\text {st }}$ gear, $8.6 \mathrm{~kW} @ 10 \mathrm{~km} / \mathrm{h}, 33 \mathrm{~kW} @ 60 \mathrm{~km} / \mathrm{h}$; for 2nd gear ratio, $16 \mathrm{~kW} \& 40 \mathrm{~km} / \mathrm{h}$ and $27 \mathrm{~kW} @ 100 \mathrm{~km} / \mathrm{h}$.

The electromagnetic engine or EME (Figure 3) has been made with the electromagnetic copper coils, leed switch and permanent magnet for a 2 speed gear box. The power of the electromagnetic system has been developed with keepingconstant voltage and varying current supply by a $\mathrm{LiFePO}_{4}$ battery

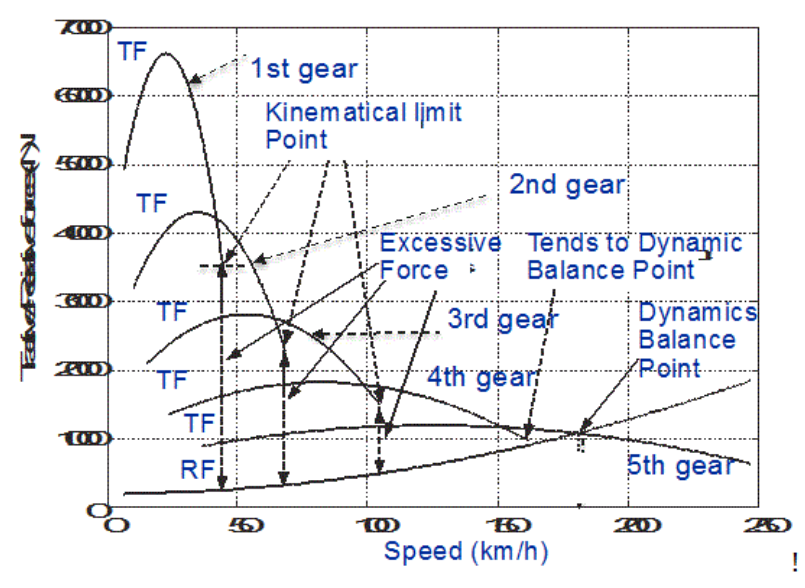

TF - Traction Force (N) ; RF - Resistive Force (N)

Figure 2: Traction force for different gear ratio.

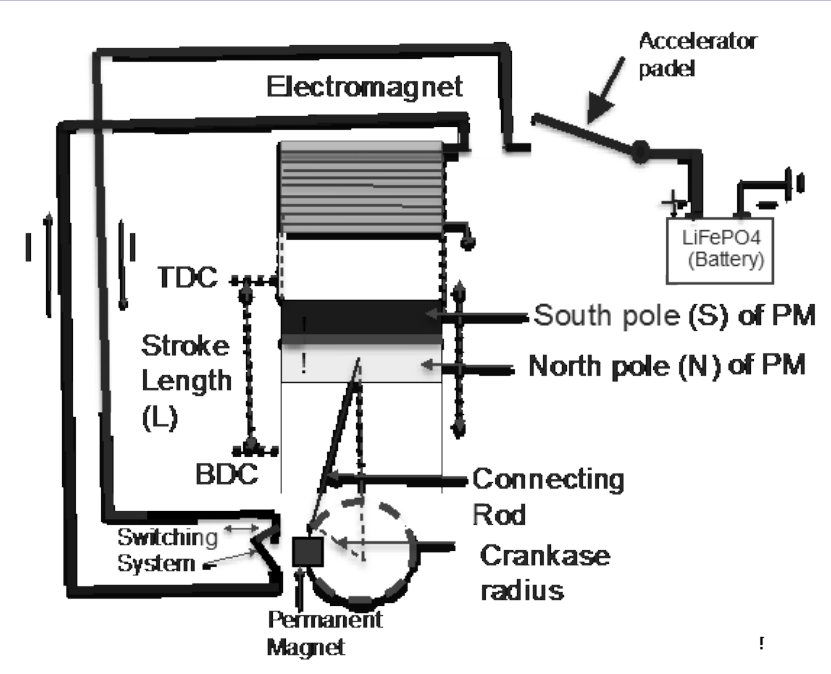

Figure 3: Schematic diagram of electromagnet.

pack of nominal voltage $48 \mathrm{~V}$ and capacity $44 \mathrm{Ah}$. The EME would be able to develop the traction torque for the vehicle as needed for the $1^{\text {st }}$ gear and $2^{\text {nd }}$ gear. The EME has able to do the pushing (or repulsing) and pulling (or attracting) the piston with the magnetic field as soon as power supply to the coils, which would meet the traction power, demands of the car. The discussion in this study has been emphasized on pushing mechanism, as it is more crucial part to maintain desired torque ratio than the pulling one. The actuator solenoid has been designed to develop the maximum electromagnetic force to overcome the maximum traction force. Figure 3 shows the electromagnetic engine.

The mathematical representation are developed for the Electromagnetic Actuation (EMA) mechanism by considering the dynamic behaviour of the magnetic flux, density, strength, electromagnetic force and energy according to the Faraday's Law, Ampere's Law and Lenz's law, Maxwell's dynamic condition, and the modified equations (Hayt \& Buck, 2006). The design criteria of the electromagnetic engine are the electromagnetic force, which would meet the vehicle traction force requirement.

The total electromagnetic force (emf), $\mathrm{V}_{\mathrm{emf}}$ at the magnetic field of current conducting solenoid is given by the general equation [11],

$$
V_{e m f}=-N \frac{d}{d t} \int_{s} B \sin \omega t d s
$$

Where, $F_{e m}$ is the electromagnetic force for the total solenoid coil length, $L_{\text {wire }}=a \int d \phi, B$ is the magnetic flux density for whole solenoid, $I$ is the current passing through the conductor wire around EMA. It is noted that the properties of EMA depends on the: geometry of the magnetic core, amount of air gap in the magnetic circuit, core material and solenoid temperature. In the geometry of magnetic core the number of turns is considered as circular loop although the turns are slightly helical in shape.

So the magnetic flux density (B) along the $z$ direction for the segment of wire in a single winding (Figure 4) is: 


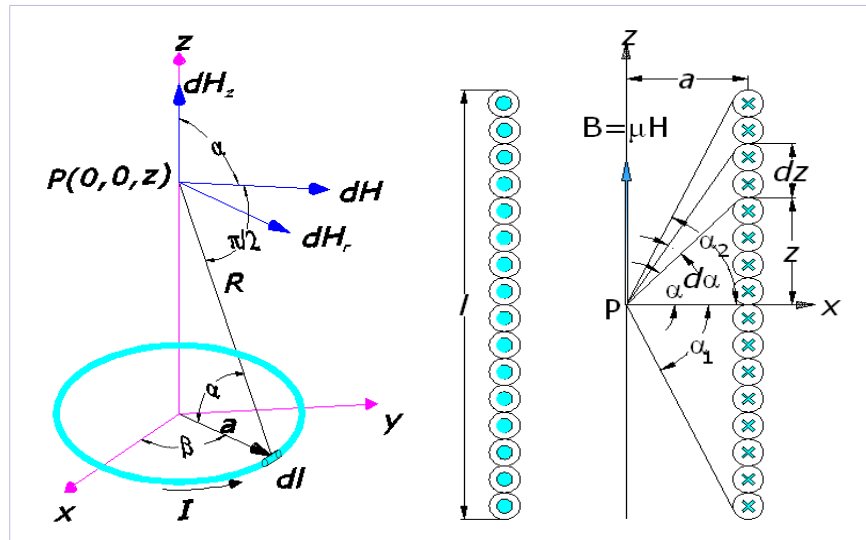

Figure 4: Magnetic field of single and multiple coil winding

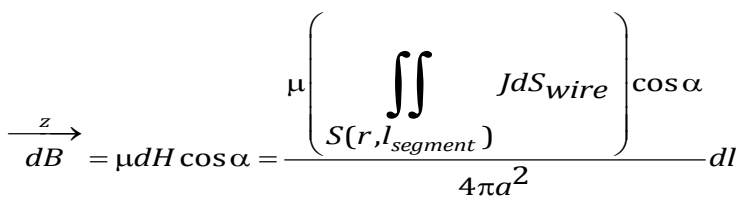

where, $\mu$ is magnetic permeability, $\mathrm{dH}$ magnetic flux intensity along the $\mathrm{z}$ direction $\alpha$ is the angle between resultant magnetic flux intensity and $\mathrm{z}$ axis. $\mathrm{J}$ is the current density across wire segment cross section $S_{\text {wire, }}$ a is the radius of the wire segment from the centre of the solenoid and $\mathrm{dl}$ is the length of the wire segment split. The magnetic concentration is gathered in the centre of the solenoid P. Therefore, overall magnetic flux density can be presented as:

$$
\begin{aligned}
& \vec{B}_{Z}=\hat{z} \sum_{a=\frac{h_{1}}{2} h_{z=-}}^{h_{2} / 2} \sum_{L_{\text {solenoid }} / 2}^{L_{\text {solenoid }} / 2}\left(\frac{\mu\left(\iint_{S\left(r, l_{\text {segment }}\right)} J d S_{\text {wire }}\right)}{4 \pi \sqrt[3]{a^{2}+z^{2}}} \int_{I(a . \beta)} d I\right) \\
& \left.=\hat{z} \sum_{a=\frac{h_{1} / 2}{h_{2}} 2} \sum_{z=-}^{L_{\text {solenoid }} / 2} / \frac{\mu\left(\iint_{S\left(r, l_{\text {segment }}\right)} J d S_{\text {wire }}\right) a^{2}}{2 \cdot \sqrt[3]{a^{2}+z^{2}}}\right)
\end{aligned}
$$

where, $h_{2}, h_{1}$ is the outer and inner radius of the solenoid from the centre $P$. $L_{\text {solenoid }}$ is the length of the solenoid. It is noted that the development of $F_{e m}$ is the function of supplied current (i.e. $F_{\text {em }}=f^{n}\left(\int_{s\left(r, l_{\text {segment }}\right)} J d S_{\text {wire }}\right)$ ). Thez supplied current of the solenoid is controlled with the controlling the voltage for the desired $F_{e m}$. The main purpose of controlling current is to prevent the actuator from temperature spike. Figure 4 shows that the magnetic flux (B) develops with supplying current to each loop of the EMA solenoid. The magnetic flux squeezes to the solenoid $x$ direction and expands it along the $z$ direction. The largest $F_{e m}$ is generated in the middle of the solenoid due to magnetic field concentration which causes the plunger to attract (push) and to repel (push).

Simplifying Equation (5) the total $B$ at point $P$ can be estimated by using the equation of [11]:

$$
\begin{aligned}
B & \left.=\hat{z} \sum_{a=\frac{h_{1} / 2}{h_{2}} / 2}^{L_{\text {solenoid }} / 2} \sum_{z=-L_{\text {solenoid }} / 2}^{\mu}\left(\frac{\iint_{s\left(r, I_{\text {segment }}\right)} J d S_{\text {wire }}}{2 \cdot \sqrt[3]{a^{2}+z^{2}}}\right) a^{2}\right) \\
& =\hat{z} N_{\text {loop }} \frac{\mu N_{\text {wind per length } I}}{2 L_{\text {solenoid }}}\left(\sin \alpha_{2}-\sin \alpha_{1}\right)
\end{aligned}
$$

Where, $\mu$ is magnetic permeability (degree of magnetization of a material in response to magnetic field), $N_{\text {wind per length }}$ is the number of turns in a single loop, $N_{\text {loop }}$ is the number of loop in solenoid housing, $N=N_{\text {wind per length }} . N_{\text {loop }}$ is the total number of turns in the solenoid. $I=\int_{S\left(r, l_{\text {expmer }}\right)} J d S_{\text {wire }}$ is the total supply current, $L_{\text {solenoid }}$ is the solenoid length and ${ }_{\alpha=\tan ^{-1}}\left[\frac{L_{\text {solamad }} / 2}{\frac{a}{2}}\right]$ is the limiting angle with radius $(a)$ for the first loop of the solenoid depends on solenoid inner dimension $\left(h_{1}\right)$. Therefore, the electromagnetic force:

$$
F_{e n}=N \frac{\mu I^{2}}{L_{\text {solenoid }}}\left(\sin \alpha_{2}-\sin \alpha_{1}\right) l_{\text {sin gle winding }}
$$

If the solenoid length is much larger than its radius, then $\alpha_{2} \cong 90^{\circ}$ and $\alpha_{1} \cong(-) 90^{\circ}$ in which Equation (7) reduced to:

$$
F_{e n}=N \frac{\mu I^{2}}{L_{\text {solenoid }}} l_{\text {sin glewinding }}=N \frac{\mu I^{2}}{L_{\text {solenoid }}} 2 \pi a
$$

Boundary conditions of the electromagnetic force assisted to figure out the maximum I while a is the radius of the wire segment from the centre of the solenoid and $\mathrm{L}_{\text {solenoid }}$ is the length of the solenoid are assumed to be defined and play around $\mathrm{N}$ for satisfying with desired magnetic force.

\section{Electromagnetic Energy}

The electromagnetic energy that has been developed in EMA due to the movement of electric charges. The electromagnetic energy stored in the solenoid $E_{\text {eng }{ }^{\prime} L i}$ due to its inductance can be estimated by using the following equation: (Rahman, et al. [12]):

$$
\begin{aligned}
E_{\text {eng. } L_{i}} & =\frac{1}{2} \mu \frac{N^{2} A_{\text {solenoid }}}{L_{\text {solenoid }}}\left(\frac{B \text { solenoid }}{\mu N}\right)^{2}=\frac{1}{2} \frac{B^{2} L_{\text {solenoid }} A_{\text {solenoid }}}{\mu} \\
& =\frac{1}{2} \frac{B^{2}}{\mu} V_{\text {ol }}^{m}
\end{aligned}
$$

Where, Vol $_{\text {solenoid }}^{m}$ is the volume of the interior of the solenoid, with, $V o l_{\text {solenoid }}^{m}=L_{\text {solenoid }} A_{\text {solenoid }}, L_{\text {solenoid }}$ is the length of the solenoid, $A_{\text {solenoid }}$ is surface of the coil or cross-sectional area of the solenoid loop. 


\section{Electromagnetic Energy Loss}

Electromagnetic energy loss of the EME is assumed to loss due to irreversible loss (magnetic hysteresis loss), temperature spike (eddy current loss) and the magnetic flux leaking through the solenoid surface. First two different kinds of energy loss is representing as core loss. An element of copper wire is considered as shown in Figure 3 in order to identify the each segment loss and later on the total summation of the segments for a winding loss is followed. Multiplication of this value with total number of winding presents the total loss in solenoid actuator.

Eddy current loss: The eddy current loss is caused by the currents induced in the magnetic material due to the with the magnetic induction. Indeed, the time varying flux $\varphi$, enclosed by each loop in the magnetic material, induces an electromotive force due to the electric conductivity $\sigma\left(5.8 \times 10^{7} \mathrm{~S} / \mathrm{m}\right.$ at $20{ }^{\circ} \mathrm{C}$ for copper) of the magnetic material, eddy currents are generated. This generation accelerates as soon as move farther apart from the centre of wire element. The magnetic field in the magnetic elements leads to the eddy current losses during a time interval Total eddy current loss can be estimated by using the equation (Dupre, et al. [13]):

$$
\begin{gathered}
E_{\text {eddycurrent loss }}=N \int_{t_{1}}^{t_{2}}\left(\sum_{\text {no.segment }}\left(\int_{y=0}^{l_{\text {segment }}} \int_{\theta_{s}=0}^{2 \pi} \int_{x=Z=0}^{2 r} \frac{J^{2}}{\sigma} r \cdot d r . d \theta \cdot d y\right)\right) d t \\
=N \frac{\pi r^{2} I^{2} l_{\text {sin }} \text { glewinding }}{\sigma}\left(t_{2}-t_{1}\right)
\end{gathered}
$$

where, $N$ is the total number of winding in solenoid, $J$ is the current density, $I$ is the supply current, $r$ is the wire radius, $l_{\text {single }}$ winding is length of single winding, $\sigma$ is the electric conductivity and $\left(t_{2}-t_{1}\right)$ is the time interval of the solenoid operation. The induction of eddy currents within the core causes generation of heat. Lamination of the core material can reduce eddy current loss. Besides, there is also movement of magnetic domains. As the magnetic field changes, some magnetic domains grow while others shrink, thus the walls of the domains can be said to move. This movement absorbs energy. Circulating eddies of current have inductance and thus induce magnetic fields. These fields create opposite effect on acting electromagnetic forces.

Hysteresis loss: Hysteresis is well known in ferromagnetic materials. When an external magnetic field is applied to a ferromagnetic material, the atomic dipoles align themselves with the external field. Even when the external field is removed, part of magnetic alignment will be retained. The hysteresis loss is related the magnetic induction $\mathrm{B}$ and the magnetic field $\mathrm{H}$ in the material depend on the history of the magnetic field. For the time interval from $t_{1}$ to $t_{2}$, the hysteresis loss can be computed by using the equation [Dupre, et al. 1996]:

$$
\begin{gathered}
E_{\text {hysteresisloss }}=N \sum_{\text {no.segment }}\left(\int_{y=0}^{l_{\text {segment }}} \int_{\theta_{s}=0}^{2 \pi} \int_{x=Z=0}^{r}\left(\int_{H\left(t_{1}\right)}^{H\left(t_{2}\right)} H \frac{\partial B}{\partial H} d H\right) \cdot r . d r . d \theta \cdot d y\right) \\
=N \frac{\pi r^{2}\left(B_{t_{2}}-B_{t_{1}}\right)^{2} l_{\text {sin }} \text { glewinding }}{2 \mu}
\end{gathered}
$$

where, $N$ is total number of winding in solenoid coil, $H$ is magnetic field intensity, $B$ is the magnetic flux density with respect time, $r$ is the wire radius, $l_{\text {single winding }}$ is the length of single winding and $\mu$ is the magnetic permeability.
Electromagnetic flux leaking loss: Electromagnetic flux leaking through the surface of the solenoid results significant loss of electromagnetic field when there is no shield with the solenoid surface. Outer loop windings are only considered in this study for finding the losses due to leakage. The magnetic field loss due to flux leaking of the solenoid can be computed by using the simplified equation [11]:

$$
H_{i n}=\frac{r_{i n} \int_{S\left(r_{i t}, l_{\text {segment }}\right)} J d S_{\text {wire }}}{2 \pi r^{2}} \quad\left(\text { for } r_{\dot{n}} \leq r\right)
$$

$$
\begin{aligned}
& \text { Again, } \quad \int_{\text {out }} J d S_{\text {wire }} \\
& 2 \pi r_{\text {out }}
\end{aligned} \quad\left(\text { for } r_{\text {out }}>r\right)
$$

$H_{\text {out }}$ is the main concern for finding the flux leakage through the solenoid surface. The magnetic field $H_{i n}$ is effective to develop the desired magnetic force to operate the movable sheave of the pulley while $H_{\text {out }}$ is the causes of the magnetic loss. The energy loss of the solenoid due to the flux leaking can be computed by the simplified equation [11]:

$$
\begin{gathered}
E_{\text {flux leakage loss }}=N_{\text {out }}\left(\sum_{\text {no.segment }} \frac{S\left(r, l_{\text {segment }}\right)}{2 \pi r_{\text {out }}} J / 2 d S_{\text {wire }}\right. \\
\left.=\frac{I\left(h_{2}-h_{1}\right) L_{\text {solenoid }}}{4 \pi r_{\text {wire }} r_{\text {out }}} l_{\text {sin glewinding }}\right)
\end{gathered}
$$

where, $\mathrm{N}_{\text {out }}$ is number of winding in the solenoid surface. J/2 is due to the half circle of the copper wire, I is the supply current. $h_{2}$ and $h_{1}$ is the solenoid outer and inner diameter respectively, $\mathrm{L}_{\text {solenoid }}$ is length of the solenoid, $l_{\text {single winding }}$ is length of single winding $r_{\text {wire }}$ is the wire radius and $r_{\text {out }}$ is the outer radius where $\mathrm{r}_{\text {out }}>\mathrm{r}_{\text {wire }}$.

\section{Development Of Electromagnetic Engine}

The cylinder block is designed with enough space for coiling the copper wire, which generates electromagnetic force to develop the effective torque at the crankshaft as soon as current supply. It react the ferromagnetic material such as steel, iron and all types of permanent magnet. Then, the piston is ensured the linear movement of the part due to the presence of electromagnetism effect. A connecting rod is a part of an engine that exchanges movement from the cylinder to the crankshaft and functions as a lever arm. Connecting rods are produced using cast aluminium alloy and are intended to withstand dynamic anxieties from burning and inside-cylinder motion development. However, the EME engine model, non-magnetic and non-conductivity material is used to reduce the weight of the model and to make the electromagnetic force generated easy to attract the piston inside out. The little end of the connecting rod connects with the piston 
with a type of locking which known as piston pin. The piston pin or also known as wrist pin gives a turning point between the piston and connecting rod. A usual type of bolt-and-nut lock is utilized to hold the piston pin set up. The power from the linear movement of the piston is transmitted to the crankshaft. Crankshaft is a type of shaft that obtained sequential motion and change it into circular motion, which produces the propelling force of the vehicle. Figure 5 shows the electromagnetic engine model, which is made just for understanding if the EME operational principle shows the same principle of Internal Combustion Engine (ICE).

The switching mechanism is the most tricky and critical part of this engine model (Figure 6). The correct positioning of the Reed switch at the engine model specifically at the flywheel rotation will certify the maximum power generated as the current is supplied. One end of the flywheel is mounted with a Neodymium permanent magnet to be reacted with the Reed switch. The meeting of the magnet and the Reed switch is assured at the position where the crankshaft at its lowest position of piston which has the highest potential energy. As an external force is given to the flywheel, the magnet will eventually meet and respond to the switch which creating a spark and complete the circuit. Thus, the copper coil is magnetized and the piston moves inside the cylinder in a second. As magnet is away from the switch and the circuit is opened (no current supplied), the wheel is still in rotation by the inertia it creates. The piston will move backward. This motion is repeated again and again and the switch is closed and opened in accordance to the permanent magnet position. Hence, a rotational motion is obtained as the piston moves back-and-forth smoothly.

\section{Result and Discussion}

The engine model cannot be operated smoothly if the voltage supply is less than $10 \mathrm{~V}$. Thus, in the testing of the engine, the voltage used is from $10 \mathrm{~V}$ until $20 \mathrm{~V}$. Figure 7 indicates that as the current is increased in the circuit, the electromagnetic force that actuates the piston is also in increasing manner. The maximum force is $2.113 \mathrm{~N}$ at the amount of current $0.81 \mathrm{~A}$ while the minimum force is $1.526 \mathrm{~N}$ at current value of $0.45 \mathrm{~A}$. Figure 8 shown is the relationship between the current rating in the circuit and the rotational speed of the wheel. It has a linear relation between both of the variables. The highest RPM that it generates when $0.81 \mathrm{~A}$ current flows in the circuit is 517.7RPM. The lowest RPM is $271.6 \mathrm{RPM}$, which is reflected by $0.45 \mathrm{~A}$ of current.

The speed of EME has been realized applying loads in the range of $0.01 \mathrm{~kg}$ to $0.06 \mathrm{~kg}$ at the crankshaft by using a small pulley. The speed of rotation of the wheel decreases and when the load is increased to a higher value the speed becomes lower. This experiment also shows that the electromagnetic force can overcome any load under its capacity. The capacity of this motor is based on its own configuration and configuration which includes the number of turns of the copper coil and the voltage supplied to the coil. Therefore, Figure 9 shows the performance of the EME model under different loads. The efficiency of the EME model is shown in Figure10. The result shows that when the input power is increased, the efficiency continues to increase

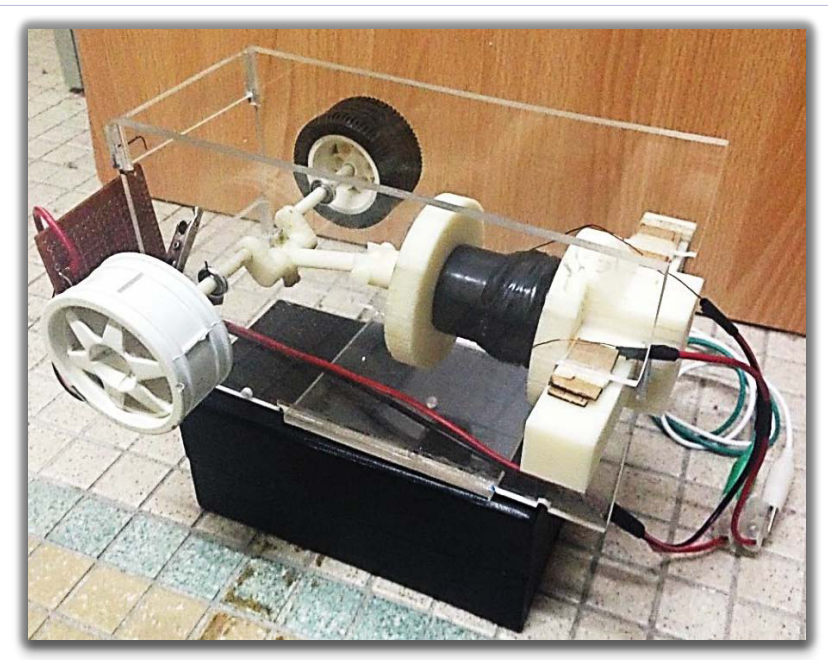

Figure 5: Model of Electromagnetic Engine.

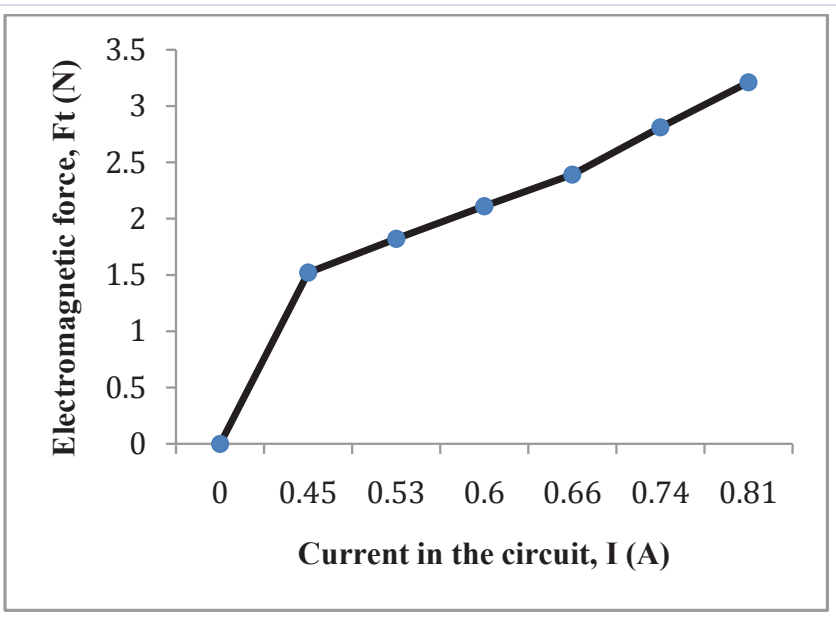

Figure 6: Switching mechanism.

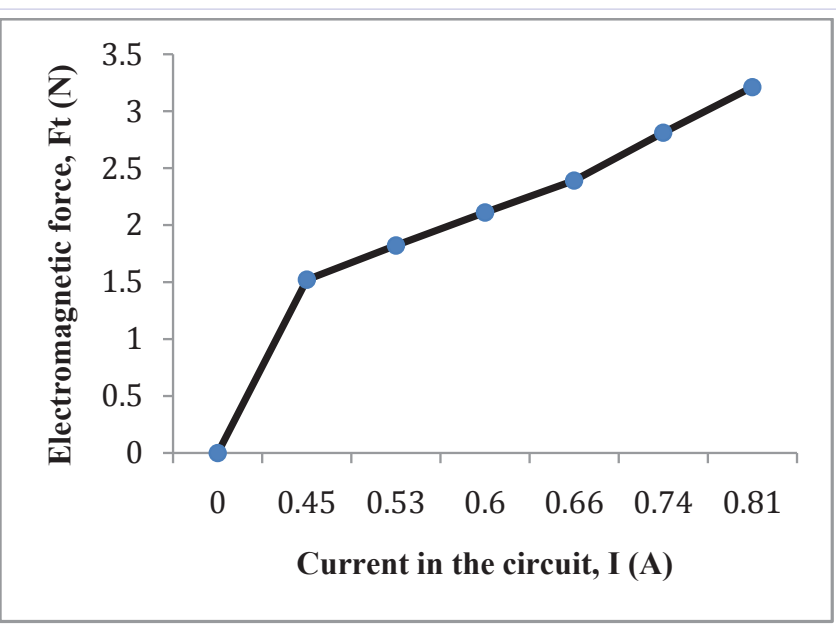

Figure 7: Electromagnetic force exerted in the cylinder 
but in small value. The efficiency obtained from the experiment is relatively lower than the efficiency of conventional engines, while it must be higher than the engines. The lower efficiency has been obtained due to the too many losses that occur in the configuration of the circuit and the configuration of the components. Magnetic flux leakage is occurring on the solenoid coil as there is no shielding. The heat generated in the coil when voltage is supplied has caused the loss of power.

The thin and thin type of copper wire is one of the factor making the coil easily heated by the current flow. In addition, friction losses also existed in the components of the engine model. It has taken place enormously in the movement between the piston and the cylinder wall. It prevents piston movements linearly in the hole and reduces the rotational speed of the crankshaft. The possible cause of this situation is the misalignment of the configuration of the model. It could be concluded that many electromagnetic forces have been used to overcome friction. Rahman et al., 2015, have reached the same conclusion about the development of the electromagnetic actuator for the CVT operation. It is expected

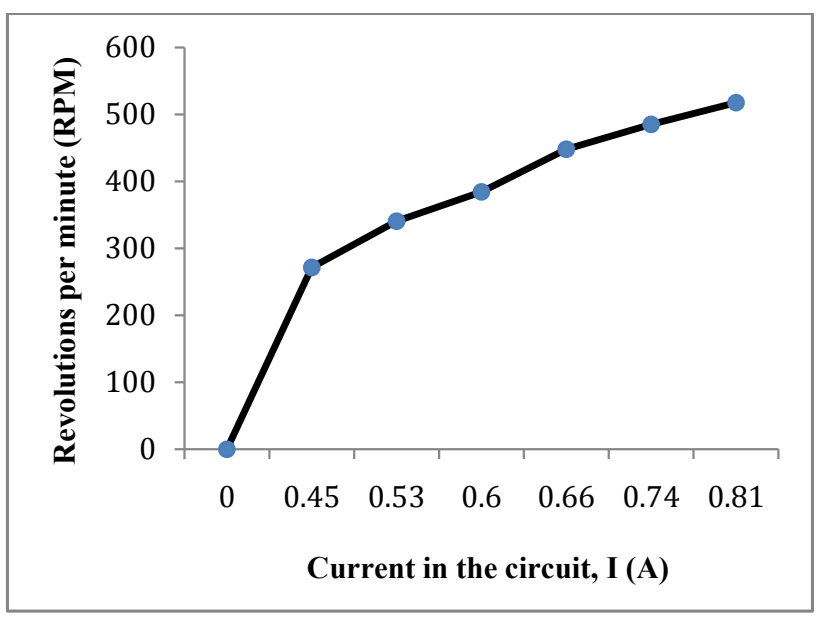

Figure 8: EME's RPMs vs. Current Supplied.

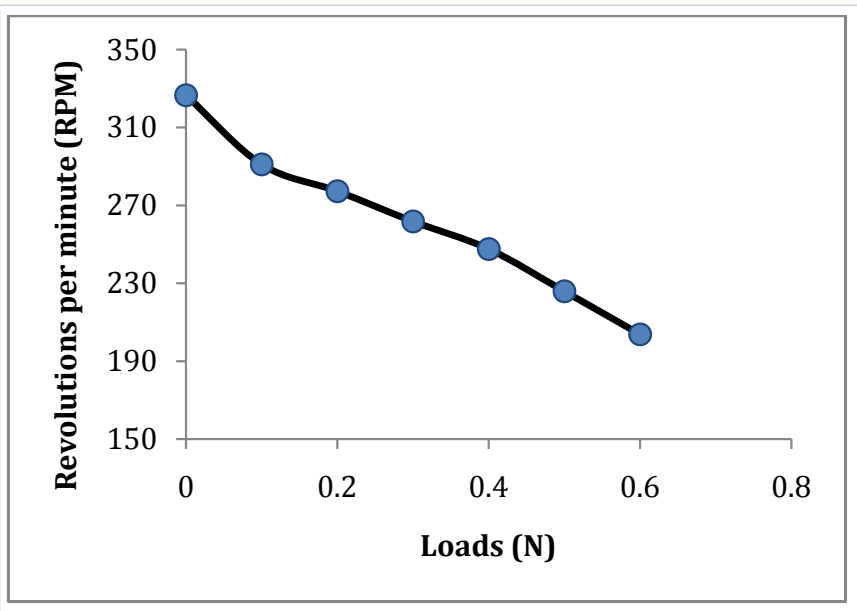

Figure 9: EME’s RPMs vs. Loads applied.

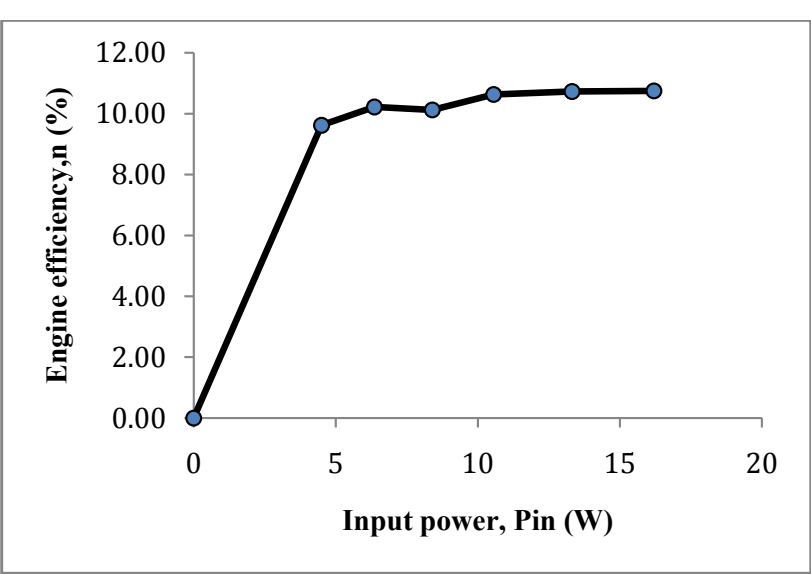

Figure 10: Engine efficiency vs. Input power.

that the EME efficiency could be around $35-40 \%$ by properly sealing the magnetic flux of the electromagnet, correct alignment, exact adjustment of the piston and the cylinder. The development of piston and cylinder with aluminum alloy and metallic coating could improve EME efficiency by about 10\%. In general, EME performance could be around $40-45 \%$, which would be higher than the conventional ICE.

\section{Conclusion}

The electromagnetic engine for the vehicle used has many advantages when compared to existing IC engines. It has also a few added values against the motor that is used in the electric vehicle such as easy to handle. The piston is located in a leading hole of a holder and electromagnetic solenoid windings are fixed at the upper end of the cylinder hole. Hence, while engaging the reciprocal motion, the magnetized piston is being held in position as guided by the leading hole. On the other hand, the Leed switch is the main component of all. If compared to IC engines, the function of the switch is similar to the distributor that controls the correct firing order of the spark plug. The EME performance could maintain in the expected range by replacing the permanent magnet over a period of regular interval. The power source of this electromagnetic engine is only from the battery. By designing the battery pack with LiFePO4, the EME performance could be enhanced. Thus, it makes this type of engine not as flexible as IC engines that have been used in today's vehicles on the road.

\section{References}

1. Ayman AA, El-Shafei Z, Hamed A, Salem F. An Antilock-Braking Systems (ABS) Control: A Technical Review. ICA. 2011;2:186-195.

2. James $\mathrm{R}$ Powell. 2002. Available from: http://www.fi.edu/ winners/2000/powell_james.faw?winner_id=3708

3. James P, Danby G. Maglev the new mode of transport for the 21st century. The 21st Century Science and Technology Magazine. 2005. Available from: http://www.21stcenturysciencetech.com/articles/ Summer03/maglev2.html

4. Bonsor K. How Maglev trains work. 2010. Available from: http:// science.howstuffworks.com/transport/engines-equipment/maglevtrain 1.htm 
5. Byers DC. The NASA electric propulsion program. JSASS/AIAA/DGLR 17th International Electric Propulsion Conference. 1984:1-9.

6. Pulatov V. Magnetic propulsion systems. Progress in Aerospace Sciences. 2001;37(3):245-261.

7. Behrooz M, David C. Vehicle Powertrain Systems. 1st Edition. John Wiley \& Sons, Ltd; 2012.

8. Hurley WG, Wolfie WH. Electromagnetic Design of a Magnetic Suspension System. IEEE Transactions on Education. 1997;40(2):124130.

9. Jayaprakash A, Balaji G, Bala Subramaniam S, Naveen N. Studies on Electromagnetic Engine, Department of Mechanical Engineering, Vel Tech Engineering College, Chennai, Tamilnadu, India. 2014.

10. Parlikar TA, Chang WS, Qiu YH, Seeman MD, Perreault DJ, Kassakian JG, Keim TA. Design and Experimental Implementation of an Electromagnetic Engine Valve Drive, IEEE/ASME Transactions on Mechatronics, 2005;10(5):482-494. DOI: 10.1109/ TMECH.2005.856221
11. Ulaby FT. Electromagnetics for Engineers. Pearson International Edition. Pearson Education, Inc Upper Saddle River, NJ. 2005.

12. Rahman A, Sharif SB, Mohiuddin AKM, Rashid M, Hossain A. Energy efficient electromagnetic actuator for CVT system. Journal of Mechanical Science and Technology. 2014;28(4):1153-1160.

13. Dupre LR, Van RK, Melkebeek JAA. On a magnetodynamic model for the iron losses in non-oriented steel laminations. J Phys D:Appl Phys. 1996;29(3):855-861.

14. Munro John. A Trip to Venus. London: Jarrold \& Sons; 1897. 26-28.

15.Xiaoming Z, Wenfang X, Suong VH, Rui Z. Design and Analysis of Collaborative Automated FiberPlacement Machine.IJARA.2016;1(1):114. DOI: http://dx.doi.org/10.15226/2473-3032/1/1/00105 